\title{
Higher Order Cross-Correlation Functions from Galaxy-Galaxy-Galaxy Lensing
}

\author{
Peter Watts ${ }^{1}$ and Peter Schneider ${ }^{1}$
}

${ }^{1}$ Institut f. Astrophysik u. Extr. Forschung, Universität Bonn, Auf dem Hügel 71, D-53121

Bonn, Germany. Email: pwatts@astro.uni-bonn.de (PW), peter@astro.uni-bonn.de (PS).

\begin{abstract}
Galaxy-galaxy lensing (GGL) measures the 2-point cross-correlation between galaxies and mass in the universe. In this work we seek to generalise this effect by considering the third-order correlations between galaxies and mass: galaxy-galaxy-galaxy lensing. We introduce the higher-order cross-correlation functions and discuss their physical interpretation in terms of the halo model. We then demonstrate that measuring the lensing shear of background galaxies about foreground galaxy pairs, which we term galaxy-galaxy-galaxy lensing, provides a measure of the third-order cross functions. We note that current surveys in which detections of the cosmic shear 3-point correlation function have been reported should also be able to detect galaxy-galaxy-galaxy lensing. Indeed, we suggest that this effect may already have been detected in recent studies of GGL.
\end{abstract}

\section{Introduction}

Galaxy-galaxy lensing (GGL) is the shear distortion of background galaxies by individual foreground galaxies. It offers the possibility of allowing us to study the mass environments of galaxies of particular colour, type or luminosity, their occupation of dark matter halos and their relationship to the large-scale structure of the universe. Such relations together make up the the so-called "bias" between galaxies and dark matter. The measurement of GGL was first attempted by Tyson et al. (1984), and its first detection was reported by Brainerd et al. (1996). Since then a number of GGL measurements have been reported: in the HST Medium Deep Survey (Griffiths et al. 1996), the Hubble Deep Field (Dell'Antonio \& Tyson 1998; Hudson et al. 1998), and more recently, in the RCS survey by Hoekstra et al. (2001, 2002), the COMBO-17 survey (Kleinheinrich et al. 2004) and the Sloan Digital Sky Survey (Fischer et al. 2000; McKay et al. 2001; Guzik \& Seljak 2002; Sheldon et al. 2004, Seljak et al. 2004).

The basic observable of GGL is the 2-point (cross) correlation function (2PCF) between galaxies and dark matter. Such a quantity is easily generalisable to higher-order so that one may define sequence of $n$-point cross-correlation functions. In this paper we consider the meaning of such higher-order statistics and demonstrate how they may be detected through observations similar to those of standard galaxy-galaxy lensing. We argue that the next generation of cosmic shear surveys, which will measure the 3-point correlation function $(3 \mathrm{PCF})$ of the cosmic shear field, will also be in an ideal position to measure the three-point cross-correlation functions. Indeed, we point out that detection of higherorder cross-correlations may have already been made in existing studies of GGL.

\section{Definitions}

We begin with some basic definitions in terms of continuous density fields. The overdensity of dark matter $\left(\delta_{\mathrm{d}}\right)$ and of galaxies $\left(\delta_{\mathrm{g}}\right)$ can be defined at comoving position $\mathbf{x}$ 
in the following way:

$$
\delta_{\mathrm{d}}(\mathbf{x})=\frac{n_{\mathrm{d}}(\mathbf{x})}{\bar{n}_{\mathrm{d}}}-1, \quad \delta_{\mathrm{g}}(\mathbf{x})=\frac{n_{\mathrm{g}}(\mathbf{x})}{\bar{n}_{\mathrm{g}}}-1 .
$$

We mean by $n_{\mathrm{d}}(\mathbf{x})$ and $n_{\mathrm{g}}(\mathbf{x})$ the number density of dark matter particles or galaxies respectively such that the chance of finding a galaxy in the volume $d V$ is given by $n_{\mathrm{g}}(\mathbf{x}) d V$, and $n_{\mathrm{d}}(\mathbf{x}) d V$ gives the probability of finding a dark matter particle. The 2point autocorrelation function for the dark matter and galaxies are defined in the usual way by the ensemble averages

$$
\xi_{\delta \delta}\left(x_{1}\right)=\left\langle\delta(\mathbf{x}) \delta\left(\mathbf{x}+\mathbf{x}_{1}\right)\right\rangle, \quad \xi_{\mathrm{gg}}\left(x_{1}\right)=\left\langle\delta_{\mathrm{g}}(\mathbf{x}) \delta_{\mathrm{g}}\left(\mathbf{x}+\mathbf{x}_{1}\right)\right\rangle .
$$

We can define the cross-correlation function by

$$
\xi_{\delta \mathrm{g}}\left(x_{1}\right)=\left\langle\delta(\mathbf{x}) \delta_{\mathrm{g}}\left(\mathbf{x}+\mathbf{x}_{1}\right)\right\rangle,
$$

These quantities are related to their respective power spectra through the Fourier transform relation

$$
\xi_{a b}(x)=\int \frac{\mathrm{d}^{3} k}{(2 \pi)^{3}} P_{a b}(k) \mathrm{e}^{-\mathrm{ik} \cdot \mathbf{x}} .
$$

Note that $\xi_{a b}$ and $P_{a b}$ are functions of scalar arguments only due to the statistical isotropy of the universe.

We define the higher-order correlation functions in an equivalent manner so that

$$
\begin{gathered}
\zeta_{\text {ddd }}\left(\mathbf{x}_{1}, \mathbf{x}_{2}\right)=\left\langle\delta_{\mathrm{d}}(\mathbf{x}) \delta_{\mathrm{d}}\left(\mathbf{x}+\mathbf{x}_{1}\right) \delta_{\mathrm{d}}\left(\mathbf{x}+\mathbf{x}_{2}\right)\right\rangle \text { and } \\
\zeta_{\mathrm{ggg}}\left(\mathbf{x}_{1}, \mathbf{x}_{2}\right)=\left\langle\delta_{\mathrm{g}}(\mathbf{x}) \delta_{\mathrm{g}}\left(\mathbf{x}+\mathbf{x}_{1}\right) \delta_{\mathrm{g}}\left(\mathbf{x}+\mathbf{x}_{2}\right)\right\rangle
\end{gathered}
$$

denote the third-order autocorrelations, whereas

$$
\begin{gathered}
\zeta_{\mathrm{ddg}}\left(\mathbf{x}_{1}, \mathbf{x}_{2}\right)=\left\langle\delta_{\mathrm{d}}(\mathbf{x}) \delta_{\mathrm{d}}\left(\mathbf{x}+\mathbf{x}_{1}\right) \delta_{\mathrm{g}}\left(\mathbf{x}+\mathbf{x}_{2}\right)\right\rangle \text { and } \\
\zeta_{\mathrm{ggd}}\left(\mathbf{x}_{1}, \mathbf{x}_{2}\right)=\left\langle\delta_{\mathrm{g}}(\mathbf{x}) \delta_{\mathrm{g}}\left(\mathbf{x}+\mathbf{x}_{1}\right) \delta_{\mathrm{d}}\left(\mathbf{x}+\mathbf{x}_{2}\right)\right\rangle
\end{gathered}
$$

give the two different third-order cross-correlations. The Fourier transforms of these quantities are the various cross-bispectra, $B_{a b c}$ :

$$
\zeta_{a b c}\left(\mathbf{x}_{1}, \mathbf{x}_{2}\right)=\int \frac{\mathrm{d}^{3} k_{1}}{(2 \pi)^{3}} \int \frac{\mathrm{d}^{3} k_{2}}{(2 \pi)^{3}} B_{a b c}\left(\mathbf{k}_{1}, \mathbf{k}_{2},-\mathbf{k}_{1}-\mathbf{k}_{2}\right) \mathrm{e}^{-\mathrm{i} \mathbf{k}_{1} \cdot \mathbf{x}_{1}} \mathrm{e}^{-\mathrm{i} \mathbf{k}_{2} \cdot \mathbf{x}_{2}} .
$$

One could trivially continue these definitions to even higher order. However, in this paper we restrict our attention to third-order statistics.

\section{Interpretation and motivation}

In the context of GGL, the physical interpretation of the galaxy-dark matter 2PCF is relatively simple: on small scales, for isolated foreground galaxies, this function can be taken to be a direct measure of the mass distribution about individual galaxies. On larger scales one may instead think of the cross correlation function in terms of the bias and correlation parameters $\xi_{\mathrm{gd}}=b r \xi_{\mathrm{dd}}$ (Dekel \& Lehav 1999). A major application of GGL, in combination with observations the of cosmic shear correlation functions, is then to measure these bias and correlation parameters for various samples of galaxies over a range of scales (Schneider 1998, Hoekstra et al. 2002).

For the third-order quantities defined in the previous section, the physical interpretation is not clear and it is not so easy to establish precisely the information that these 
functions contain concerning the bias. One could proceed as in the case of the 2-point statistics and define a new set of third order bias parameters: these quantities would measure some element of the non-Guassianity of the bias. This approach is discussed in Schneider \& Watts (2004). Alternatively, one may use the Halo Model (Seljak 2000, Ma \& Fry 2000, Peacock \& Smith 2000) to probe the information content of the correlation functions in terms of the Halo Occupation Distribution (HOD; see Berlind \& Weinberg 2002).

According to the halo model, on small scales - such as those probed in GGL - the cross power spectrum is dominated by the 1-halo $(1 \mathrm{H})$ contribution. This is written as

$$
P_{\mathrm{gd}}^{1 \mathrm{H}}(k)=\int \mathrm{d} m n(m) \frac{m}{\bar{\rho}} \frac{\langle N \mid m\rangle}{\bar{n}_{\mathrm{g}}} \tilde{U}_{\mathrm{d}}(k \mid m)\left[\tilde{U}_{\mathrm{g}}(k \mid m)\right]^{p} .
$$

where $\tilde{U}_{d}(k)$ is the (mass normalised) dark matter halo density profile, $n(m)$ is the mass function and $\bar{\rho}$ is the background density [see Cooray \& Sheth (2001) for a review]. The radial distribution of the galaxies with respect to the dark matter is specified by the profile $\tilde{U}_{g}(k)$, while the average number of galaxies in a halo of given mass is given by the occupation number $\langle N \mid m\rangle$. The parameter $p$ takes the value 1 when $\langle N \mid m\rangle>1$, so that most halos contain at least a single galaxy, but is 0 when $\langle N \mid m\rangle<1$.

The occupation number is just the first moment of a probability distribution $P(N \mid m)$. This distribution fully specifies the population of galaxies of a particular type in dark matter halos. Together with the radial galaxy profile it makes up "two thirds" of the Halo Occupation Distribution (HOD; Berlind \& Weinberg 2002) - the remaining "third" being the velocity distribution of the galaxies with respect to the dark matter, which is not probed directly by GGL. As pointed out by Berlind \& Weinburg (2002) (see also Berlind et al. 2003), the HOD offers a prescription for bias that is essentially complete. It's empirical determination would place strong constraints on theoretical models for galaxy formation and therefore represents a major goal for cosmology.

Using the halo model one can also write down expressions for the third-order crosscorrelations introduced in the last section. Again limiting the discussion to the nonlinear, 1-halo terms we have

$$
B_{\mathrm{ggd}}^{1 \mathrm{H}}\left(k_{1}, k_{2}, k_{3}\right)=\int \mathrm{d} m n(m) \frac{m}{\bar{\rho}} \frac{\langle N(N-1) \mid m\rangle}{\bar{n}_{\mathrm{g}}^{2}} \tilde{U}_{\mathrm{g}}\left(k_{1} \mid m\right)\left[\tilde{U}_{\mathrm{g}}\left(k_{2} \mid m\right)\right]^{p} \tilde{U}_{\mathrm{d}}\left(k_{3} \mid m\right)
$$

and

$$
B_{\mathrm{ddg}}^{1 \mathrm{H}}\left(k_{1}, k_{2}, k_{3}\right)=\int \mathrm{d} m n(m) \frac{m^{2}}{\bar{\rho}^{2}} \frac{\langle N \mid m\rangle}{\bar{n}_{\mathrm{g}}} \tilde{U}_{\mathrm{d}}\left(k_{1} \mid m\right) \tilde{U}_{\mathrm{d}}\left(k_{2} \mid m\right)\left[\tilde{U}_{\mathrm{g}}\left(k_{3} \mid m\right)\right]^{p} .
$$

The motivation for studying these higher-order cross-correlations is clear from equation (3.2), where $\langle N(N-1) \mid m\rangle$ is the number of galaxy pairs in a given halo of mass $m$ : the higher-order functions measure the higher-order moments of the occupation probability $P(N \mid m)$ and depend strongly on the radial galaxy profile $U_{\mathrm{g}}$. We note that from this perspective $B_{\text {ggd }}$ is the more interesting quantity to measure, though we remark that determination of $B_{\text {ggd }}$ could also be important since it depends on the same set of physical parameters as the cross-power spectrum (equation 3.1), though with detailed dependencies that may be complimentary. In the next section we show how a trivial generalisation of GGL can be used to measure the $B_{\text {ggd }}$ cross-bispectrum. Similar arguments can also be applied to the $B_{\mathrm{ddg}}$ function, though we do not consider this in this paper (but see Schneider \& Watts 2004). 


\section{Formalism}

To detect galaxy-galaxy lensing, one measures the average tangential shear about individual foreground galaxies. In this section we compute the average tangential shear about pairs of foreground galaxies. We will show that this is related to the cross bispectrum $B_{\text {ggd }}$. In the spirit of GGL, we refer to this cross-correlation of foreground galaxy pairs with the shear distortion of background galaxies as galaxy-galaxy-galaxy lensing (G3L).

Our calculation is carried out with background metric

$$
\mathrm{d} s^{2}=c^{2} \mathrm{~d} t^{2}-a(t)^{2}\left[\mathrm{~d} \chi^{2}+r^{2}(\chi) \mathrm{d} \Omega^{2}\right]
$$

where $\chi$ is the comoving radial coordinate, $r(\chi)$ is the comoving angular diameter distance, $c$ is the speed of light and $a$ is the cosmological expansion factor.

Consider the probability of finding a galaxy in each of two small volumes $\delta V_{1}$ and $\delta V_{2}$ together with a dark matter particle in a third volume $\delta V_{3}$. The coordinates of the $\delta V_{i}$ are $r\left(\chi_{i}\right) \hat{\boldsymbol{\theta}}_{i}$, where $\hat{\boldsymbol{\theta}}_{i}$ denotes an angular position on the sky relative to the origin of coordinates, which we take to be the midpoint of the galaxy pair. The galaxies have angular separation $\psi$. The probability of this configuration is specified by the joint distribution function

$$
\delta P(1,2,3)=\delta V_{1} \delta V_{2} \delta V_{3} \bar{n}_{g}^{2} \bar{n}_{d}\left[1+\xi_{\mathrm{gg}}(12)+\xi_{\mathrm{gd}}(13)+\xi_{\mathrm{gd}}(23)+\zeta_{\mathrm{ggd}}(123)\right],
$$

where $\bar{n}_{g}$ and $\bar{n}_{d}$ are the number density of galaxies and dark matter particles respectively. We introduce the notation $\xi^{\mathrm{gg}}(12)$ as shorthand for $\xi^{\mathrm{gg}}\left(\left|r\left(\chi_{1}\right) \hat{\boldsymbol{\theta}}_{1}-r\left(\chi_{2}\right) \hat{\boldsymbol{\theta}}_{2}\right|\right)$ etc. The joint probability distribution for a galaxy pair is

$$
\delta P(1,2)=\delta V_{1} \delta V_{2} \bar{n}_{\mathrm{g}}^{2}\left[1+\xi_{\mathrm{gg}}(12)\right] .
$$

Using Bayes' rule we have that $P(3 \mid 2,1)=P(1,2,3) / P(1 \mid 2) P(2)$. We can therefore write the average number density of dark matter particles at coordinate $r\left(\chi_{3}\right) \hat{\boldsymbol{\theta}}_{3}$, given the galaxy pair, as

$$
n_{\mathrm{d}}\left[r\left(\chi_{3}\right) \hat{\boldsymbol{\theta}}_{3}\right]=\frac{\bar{n}_{\mathrm{d}}}{1+\xi_{\mathrm{gg}}(12)}\left[1+\xi_{\mathrm{gg}}(12)+\xi_{\mathrm{gd}}(13)+\xi_{\mathrm{gd}}(23)+\zeta_{\mathrm{ggd}}(123)\right] .
$$

The projection of equation (4.4) along the radial direction gives the convergence

$$
\kappa(\hat{\boldsymbol{\theta}})=\int_{0}^{\chi_{0}} \mathrm{~d} \chi \frac{g(\chi)}{a} \delta[r(\chi) \hat{\boldsymbol{\theta}}],
$$

where $g(\chi)$ is the lensing weight function

$$
g(\chi)=\frac{3}{2}\left(\frac{H_{0}}{c}\right)^{2} \Omega_{m} \int_{\chi}^{\chi_{0}} \mathrm{~d} \chi^{\prime} \frac{r(\chi) r\left(\chi^{\prime}-\chi\right)}{r\left(\chi^{\prime}\right)} p_{g}\left(\chi^{\prime}\right),
$$

with $p_{g}\left(\chi^{\prime}\right)$ the radial distribution of background (source) galaxies. If the redshifts of both foreground galaxies are known, the convergence at angular position $\hat{\boldsymbol{\theta}}_{3}$ on the sky is

$$
\begin{aligned}
\kappa\left(\hat{\boldsymbol{\theta}}_{3}\right) & =\kappa_{13}+\kappa_{23}+\kappa_{123}, \\
\kappa_{13}\left(\hat{\boldsymbol{\theta}}_{3}\right) & =\int_{0}^{\chi_{0}} \mathrm{~d} \chi_{3} \frac{g\left(\chi_{3}\right)}{a} \frac{\xi_{\mathrm{gd}}(13)}{1+\xi_{\mathrm{gg}}(12)} \\
\kappa_{123}\left(\hat{\boldsymbol{\theta}}_{3}\right) & =\int_{0}^{\chi_{0}} \mathrm{~d} \chi_{3} \frac{g\left(\chi_{3}\right)}{a} \frac{\zeta_{\mathrm{ggd}}(123)}{1+\xi_{\mathrm{gg}}(12)}
\end{aligned}
$$

where $\kappa_{23}$ is given by a similar relation to $\kappa_{13}$. 
The average tangential shear is computed in a circular aperture of radius $\theta$ centred on the midpoint of the galaxy pair. The shear is related to the average convergence within the aperture by

$$
\left\langle\gamma_{t}\right\rangle(\theta)=-\frac{1}{2} \frac{\mathrm{d} \bar{\kappa}(\theta)}{\mathrm{d} \ln \theta}
$$

where the average convergence is

$$
\bar{\kappa}(\theta)=\frac{1}{\pi \theta^{2}} \int_{0}^{\theta} \theta^{\prime} \mathrm{d} \theta^{\prime} \int_{0}^{2 \pi} \mathrm{d} \phi \kappa\left(\hat{\boldsymbol{\theta}}^{\prime}\right) .
$$

To compute these integrals of equations (4.7) we rewrite the correlation functions in terms of their Fourier transforms [equations (2.4) and (2.9]. Following Villumsen (1996), working under the plane parallel approximation and under the assumption that $\psi, \theta \ll 1$, we find that

$$
\left\langle\gamma_{t}\right\rangle(\theta)=\gamma_{13}(\theta)+\gamma_{23}(\theta)+\gamma_{123}(\theta)
$$

where

$$
\begin{aligned}
\gamma_{13} & =\frac{1}{1+\xi_{\mathrm{gg}}\left(r_{12} \psi\right)} \frac{g\left(\chi_{1}\right)}{a} \int \mathrm{d} k k P_{\mathrm{gd}}\left(k, \chi_{1}\right) \mathrm{J}_{2}\left[k r\left(\chi_{1}\right) \theta\right] \mathrm{J}_{0}\left[k r\left(\chi_{1}\right) \psi\right] \text { and } \\
\gamma_{123} & =\frac{1}{1+\xi_{\mathrm{gg}}(r(\chi) \psi)} \frac{g(\chi)}{a} \int \mathrm{d} k_{1} \mathrm{~d} k_{2} k_{1} k_{2} \int \mathrm{d} \beta B_{\mathrm{ggd}}\left(k_{1}, k_{2}, \beta\right) \\
& \quad \times \mathrm{J}_{2}\left[r(\chi) \theta f\left(k_{1}, k_{2}, \beta\right)\right] \mathrm{J}_{0}\left[r(\chi) \psi f\left(k_{1}, k_{2}, \beta\right)\right] .
\end{aligned}
$$

and where $\mathrm{J}_{0}$ and $\mathrm{J}_{2}$ are Bessel functions of the first kind. In the above expressions we have defined $r_{12}^{2}=r^{2}\left(\chi_{1}\right)+r^{2}\left(\chi_{2}\right)-2 r\left(\chi_{1}\right) r\left(\chi_{2}\right) \psi$ and $f^{2}=k_{1}^{2}+k_{2}^{2}+2 k_{1} k_{2} \cos \beta$.

Equations (4.10) and (4.11) relate the tangential shear about a pair of foreground galaxies to the cross bispectrum and power spectrum. The result is a sum of three terms: two of these, $\gamma_{13}$ and $\gamma_{23}$, arise from the usual GGL about each of the foreground galaxies (see Guzik \& Seljak 2001). The other, which we denote $\gamma_{123}$, is the intrinsic 3-point correlation. The $\gamma_{123}$ term is non-zero only if $r\left(\chi_{1}\right)=r\left(\chi_{2}\right)=r(\chi)$, i.e both foreground galaxies lie at the same radial distance.

Measurement of the G3L signal from a galaxy survey should, at least in principle, be straightforward. A practical estimator can be obtained by first defining bins in $(\psi, \theta)$ space. Then, for each triplet of two foreground ground galaxies and one background galaxy falling within a bin, one sums the tangential shears of the background galaxies measured with respect to the midpoint of the galaxy pair, If only foreground pairs of galaxies with the same redshift are included in the sum, then subtracting the 2-point (GGL) contributions will leave the intrinsic 3-point cross correlation, $\gamma_{123}$.

\section{Conclusions}

In this paper we have introduced the higher order cross correlation functions between dark matter and galaxies. We have explored their physical interpretation in terms of the halo model and the halo occupation distribution. It was noted that the higher order cross-correlation functions measure the higher order moments of the occupation probability $P(N \mid m)$ and depend strongly on the radial distribution of galaxies in dark matter halos. By considering the average tangential shear about pairs of foreground galaxies we have introduced a new observational effect, galaxy-galaxy-galaxy lensing, which is a measure of the three-point galaxy-galaxy-mass cross-correlation. The G3L effect is complimentary to regular galaxy-galaxy lensing. Firstly it probes the higher order moments of the occupation probability $P(N \mid M)$, whereas GGL measures only the mean. Secondly 
the condition that $\gamma_{123}$ is nonzero only when the galaxies in pairs lie at smilar redhsifts implies that the dominant contribution to G3L comes from pairs of galaxies that reside in the same dark matter halo. G3L therefore explores a different mass regime to GGL since only those dark matter halos that contain at least two galaxies will contribute (i.e galaxy groups).

Observationally the G3L signal should not be difficult to detect. Although it is a thirdorder effect we note that it is only first order in the shear. It is therefore a more simple measure than the cosmic shear 3-point (or even 2-point) correlation function (see e.g Schneider \& Lombardi 2003). In principle surveys capable of measuring the cosmic shear 3-point correlation function should also be capable of measuring G3L, though photometric redshift information on the foreground population would be an advantage in order to unambiguously identify the intrinsic 3-point contribution. Indeed this signal, or integral measures of it, has probably been detected already in studies of GGL: McKay et al. (2002) have demonstrated that their measurement of GGL from the SDSS is stronger for foreground galaxies that are located in regions of high galaxy density. This detection provides a correlation between the GGL signal and the number density of galaxies, which is a signature of G3L.

We finally note that we have presented only one approach to galaxy-galaxy-galaxy lensing: that of measuring the average tangential shear about foreground galaxy pairs. An alternative approach is investigated in detail by Schneider \& Watts (2004) who define the three-point cross-correlation functions directly in terms of the shear and the projected galaxy number density. They then relate these to the third-order aperture mass statistics. This approach has the main advantage of avoiding the cumbersome integrals of equations (4.11).

\section{References}

Berlind, A. \& Weinberg, D., 2002, ApJ 575, 587

Berlind, A.A., Weinberg, D.H., Benson, A.J., Baugh, C.M., Cole, S., Davé, R., Frenk, C.S., Jenkins, A., Katz, N. \& Lacey, C.G., 2003, ApJ, 593, 1

Brainerd, T.G., Blandford, R.D. \& Smail, I., 1996, ApJ, 466, 623

Cooray, A. \& Sheth, R.K., 2003. Physics Reports, 372, 1.

Dekel, A. \& Lahav, O., 1999, ApJ, 520, 24

Dell'Antonio, I.P. \& Tyson, J.A., 1996, ApJ 473, L17

Fischer, P., McKay, T.A., Sheldon, E. et al., 2000, AJ 120, 1198

Griffiths, R.E., Casertano, S., Im, M. \& Ratnatunga, K.U., 1996, MNRAS 282, 1159

Guzik, J. \& Seljak, U., 2001, MNRAS 321, 439

Guzik, J. \& Seljak, U., 2002, MNRAS 335, 311

Hoekstra, H., van Waerbeke, L., Gladders, M.D., Mellier, Y. \& Yee, H.K.C., 2002, ApJ 577, 604

Hoekstra, H., Yee, H.K.C. \& Gladders, M.D., 2001, ApJ 558, L11

Hudson, M.J., Gwyn, S.D.J., Dahle, H. \& Kaiser, N., 1998, ApJ 503, 531

Kleinheinrich, M., Rix, H.-W., Erben, T. et al., 2004, A\&A submitted (also astro-ph/0404527)

Ma, C., Fry, J.N., 2000. ApJ, 543, 503.

McKay, T.A., Sheldon, E.S., Racusin, J. et al., 2001, astro-ph/0108013

Peacock, J.A. \& Smith, R.E., 2000. MNRAS, 318, 1144.

Seljak, U., 2000., MNRAS, 318, 203.

Seljak, U., Makarov, A., Mandelbaum, R. et al. 2004, astro-ph/0406594

Sheldon, E.S., Johnston, D.E., Frieman, J.A. et al. 2004, AJ 127, 2544

Schneider, P., 1998, ApJ 498, 43

Schneider, P. \& Lombardi, M., 2003, A\&A, 397, 809

Schneider, P. \& Watts, P.I.R., 2004, A\&A submitted

Tyson, J.A., Valdes, F., Jarvis, J.F. \& Mills Jr., A.P. 1984, ApJ 281, L59

Villumsen, J.V., 1996, MNRAS, 281, 369 